\title{
CHRONIC ORAL INFECTIONS *
}

THOMAS L. GILMER, Sc.D., M.D.

CHICAGO

In 1891 Miller, in the preface to a series of articles ${ }^{1}$ entitled "The Human Mouth as a Focus of Infection," says :

During the last few years the conviction has grown continually stronger among physicians, as well as dentists, that the human mouth as a gathering-place and incubator of pathogenic germs performs a significant rôle in the production of varied disorders of the body, and that if many diseases whose origin is enveloped in mystery could be traced to their source, they would be found to have originated in the oral cavity.

Miller isolated fifty-eight varieties of microorganisms from the mouth, many of which are pathogenic or may under favorable circumstances become so." These findings were verified by Black, Vincentini and others. ${ }^{3}$

When it is realized that in some or all mouths there may be found at times bacteria of most infections, the importance of the study of the mouth as a focal cause of disease becomes apparent. For instance, tubercle bacilli are found in the mouths of non-tuberculous patients. When the cervical glands are found to be tuberculous there can be little doubt but that the bacilli often reach the glands from the mouth through alveolar abscess, the tonsils, or through any suppurating focus in the mouth.

The prevalence of tubercle bacilli in the mouths of those not tuberculous is indicated by the experiments of Gilberti. ${ }^{4}$ He injected guineapigs, previously subjected to the tuberculin test, with mixtures of inguinal and also of cervical glands taken from children dead of non-tuberculous disease, so far as known. Of thirty animals injected with the cervicalgland mixture, eleven died from tuberculosis. Of those injected with the inguinal-gland mixture, only two died of tuberculosis.

As a result of food wedging in between teeth malposed, or between approximating teeth having cavities of decay, or between teeth with improper restored contours, the gum septum is destroyed and deep pockets are formed. The injudicious use of crowns and bridges made as substitutes for lost teeth, and their faulty adjustment are sources of great danger, since they form pockets which harbor bacteria and are irritants to the gums, peridental membrane and alveolar process. These pockets

${ }^{*}$ Received for publication, Feb. 5, 1912.

1. Miller: Dental Cosmos, September, 1891, p. 689.

2. MacFarland: Pathogenic Bacteria, Ed. 4.

3. Bacteria of the Sputa and Cryptogenic Flora of the Mouth, London, 1897.

4. Gilberti: Medical Practice Series, 1910, iii, 313. 
constantly present granulating walls and afford good incubating centers for pathogenic and other microorganisms, both aerobic and anaerobic. Unfilled cavities of decay in teeth always harbor both saprophytic and pathogenic microorganisms; also unsterilized pulpless root canals, or root canals containing gangrenous pulps or their septic remains, may be equally prolific as incubating centers.

Many persons are mouth-breathers, either occasionally or generally, owing to stenosis from malformation or disease of the nasal fossæ. The nasal fossæ offer barriers to the passage of bacteria through their labyrinthine formation, the hairs and the agglutinating material found on their walls. The mouth offers practically no such obstructions. Those having nasal stenosis breathe largely through the mouth; as a result there are admitted to the oral cavity, pharynx and tonsils, greater numbers of bacteria than when the nose performs its function normally. There may be other causes for lesions of the tonsils, but it is highly probable that the constant passing of bacteria over them from a diseased mouth is the most potent. That bacteria may pass through healthy tonsils, without causing them to become diseased, has been demonstrated by Wood. ${ }^{5}$ Some, however, find convenient lodging places in the crypts of the tonsils, where they may remain until from some cause they become active and produce local or general infection.

\section{CHRONIC ALVEOLAR ABSCESS}

Chronic alveolar abscess is so common that few go through life without one or more such suppurations. There are two forms of this disease; that which continually or periodically discharges either into the mouth, nose, maxillary sinus, or elsewhere; and the blind abscess which never discharges through a sinus, the pus being absorbed by the granulating walls of the abscess. The former, discharging pus and bacteria as indicated, cannot but be prejudicial to health, and the latter, the blind abscess, offers greater danger. Its presence is generally unobserved by the patient and may be overlooked by the physician, even if the mouth is examined, since it may not present to the eye any clinical evidence of its presence. I think 25 per cent. a safe estimate of the percentage of jaws having suppurating cavities. That greater havoc is not wrought by such focuses of infection is due either to the fact that the dosage of bacteria and their poisons is usually insufficient, or to the fact that the normal individual becomes immune. Instances, however, are not lacking which demonstrate that such focuses of infection are instrumental in causing neuritis, neuroses and secondary infections of the eye, ear and other parts; therefore when there are manifestations of disease which may be dependent on some local focus of infection, the mouth and jaws, as well

5. Cited by Sewall: Jour. Am. Med. Assn., Sept. 9, 1911, p. 869. 
as other possible sources should be examined to discover if they may not hold the key to the solution of the problem.

Cervical and submaxillary adenitis is especially common in children having diseased teeth and jaws. Frequently the glands are removed and their unsuspected cause, alveolar abscess, left untouched. Odenthal ${ }^{6}$ found glandular swellings in 99 per cent. of all children who suffered from badly decayed teeth (abscessed teeth), and only 49 per cent. in those having sound teeth.

The question which has not been answered, but must and will be, is: What is the bacteriology of chronic alveolar abscess? When this question and the question of susceptibility and immunity are answered, we can better understand the relation which these pathological cavities in the maxillæ bear to lesions in the heart, kidneys, lungs, joints, nerves and brain. Here are possibilities not to be ignored. Did similar abscesses exist in other bones of the body, their presence would demand attention, both by the patient and by the physician. Alveolar abscess is usually considered inconsequential and when the attention of the profession has been called to it, it has generally excited little interest and has been allowed to pass as an insignificant "gum boil."

Acute alveolar abscess usually manifests itself in pain, swelling, rigors and fever, but the chronic form often gives no evidence of its presence. Following the subsidence of the acute attack, the patient will often be unaware of the continuance of the condition, but unless the tooth causing it is removed, or has its pulp canal or canals disinfected and filled, the chronic form ensues and continues indefinitely, as it is rare that spontaneous cure takes place. Owing to the tortuous shapes of some root canals, it is not possible in all cases to sterilize completely and fill them to their ends, and even if the roots are rendered sterile through canal disinfection. the chronic condition is not always cured. A part of the apical portion of the root in such abscesses is often denuded of its peridental membrane by suppuration. Between this portion of the root divested of the soft tissue attachment and the adjacent granulation tissue a pocket forms which is a breeding place for microorganisms that induce continuous reinfection.

The salivary glands are doubtless occasionally infected by bacteria from the mouth. I have seen two cases of infection of the parotid gland which seemed to be traceable to an infected mouth, and several cases of infection of the submaxillary gland which could be traced to no other source.

It is but natural and proper that patients suffering from endocarditis. renal, joint and other lesions, outside the mouth, should consult the internist, orthopedist, or neurologist. On this account the oral specialist has little opportunity to tabulate cases which throw any light on the

6. Odenthal: Therapie des maladies infectieuses, p. 256. 
constitutional relation existing between the disorders before-mentioned and chronic infections in the mouth. I have frequently found marked improvement in the health of patients following the cure of diseased conditions in the mouth, but the treatment has not usually been instituted with other idea than the removal of a local lesion. The following case reports will illustrate this:

CASE 1.- Some years ago, a man, a little beyond middle life, consulted me relative to a trivial dental lesion. On making a careful examination of the entire oral cavity, I found several small sinuses discharging pus above the bicuspid teeth on one side of the upper jaw. When these openings were explored with a sharp steel probe, a large cavity was discovered in the bone, the result of alveolar abscess, the presence of which was unsuspected by the patient. On inquiring into his physical condition, I learned that for the past year he had had a cough, his digestion was impaired, and much of the time his temperature was slightly above normal. He had frequently consulted his family physician, who examined his heart, lungs, sputum, urine and blood. These gave no clue to the cause of his ill health. His appearance indicated a toxemia. I removed several teeth, curetted the abseess in the jaw and gave suitable after-treatment. The fever at once subsided, the digestion was soon much improved and the cough was lessened and finally disappeared altogether. Although seemingly the patient's physician had made a careful examination, he had overlooked one important factor, the mouth.

CASE 2.-Mrs. C., aged 30, noticed the appearance and disappearance at frequent intervals of an erythemic patch about the size of a silver quarter on the skin over the canine fossa. On examining the mouth for a possible cause for this reddened condition of the skin, I found the lateral incisor pulpless. There was no sinus; the tooth had given no trouble. The only evidence of disease found in the mouth was a slight hyperemia of the gum over the lateral incisor root indicated. The radiograph showed a pus cavity in the bone at the end of the root about the size of a large pea. Disinfection of the root did not effect a cure. I made an opening through the labial wall of the alveolar abscess, excised the end of the root and curetted the cavity. The erythemic patch on the cheek disappeared and did not return.

CASE 3.-Mr. S., aged about 25, was directed to my clinic for the treatment of a chronic abscess in the upper jaw in the vicinity of the incisors and cuspid which had proved intractable to ordinary treatment. His physical condition was much impaired, he was emaciated, his skin was sallow, his cheeks hollow, his conjunctivæ pale, eyes dull, and his lips lacked the color of health. His temperature was slightly above normal. I could elicit no history of any other illness, recent or otherwise. The patient's appearance gave the picture of a toxemia. In this case likewise the sharp steel probe revealed a large cavity in the bone, extending from the central incisor to the first molar. Oct. 20, 1911, I extracted the cuspid tooth and curetted the bone cavity. October 27, he returned to the clinic much improved. November 10 his color was normal, his eyes clear and he seemed to be well.

Did time permit, additional cases, such as Ludwig's angina, osteomyelitis and other infections and toxemias might be cited, which were due to chronic diseases of the jaws and mouth.

A casual ocular examination of the mouth is not always sufficient to demonstrate a blind alveolar abscess, since the tissues overlying the abscess may not always indicate its presence, but by careful scrutiny of the 
mouth by the eye, aided by an educated touch, a sharp steel probe and the radiograph, all such infected areas in the jaws may be discovered.

Since the internist is not usually familiar with all of the pathology of the mouth, and has not the technic and facilities for examination of the mouth and teeth, if the best results are to be obtained, it is highly important that there should be more cooperation between internists and oral specialists.

\section{PYORRHTA ALVEOLARIS}

Pyorrhea alveolaris so-called, or as better named by Black phagedenic pericementitis, is a disease strictly of the peridental membrane. It is very common; more teeth are lost from it than from caries. It is indicated by a progressive destruction of the root membrane of the tooth commencing at the gingival border, generally accompanied by flow of pus and destruction of the alveolar walls. Pus appearing at the gum margin is not always a sign of pyorrhea, as will be shown later. The clinical picture of pyorrhea simulates, though it is not identical, with conditions brought about by indiscreet use of mercury.

Simple calcic inflammation of the gingivæ due to the deposit of salivary or serumal tartar simulates pyorrhea alveolaris and is mistaken for it. If it be of the salivary type, the lime deposited is at the gum margins, on the necks of teeth, near the openings of the ducts from the salivary glands. If of the serumal type, the lime is deposited just beneath the free margin of the gums, both being productive of pus. Serumal deposits are generally found in pyorrhea pockets, but such deposits, when formed, are below the cervical margin of the peridental membrane, and the pus pockets must be there first; while serumal deposits in simple calcic inflammation of the gums are found under the gum margin, but above the occlusal attachment of the membrane. In addition to the physical appearances in pyorrhea, in extreme cases, there is always present a peculiar odor which is distinctive. These are the chief characteristic differences between pyorrhea and calcic inflammation of the gums. The removal of all deposits, in simple calcic gingivitis and calcic pericementitis, with good hygiene afterward, usually suffices for a cure, if the removal has not been too long delayed.

The etiology of pyorrhea alveolaris is unknown. It has been attributed to gout, tuberculosis, syphilis, bacteria and other causes. Bacterial examination has often been resorted to in seeking a clue to the cause of the disease and fungi have been found by Black, Miller and Archovey, which are supposed to bear a causative relation to the disease, ${ }^{7}$ but neither has been able to prove the specificity of any one organism.

7. Black: American System of Dentistry. Miller: Microorganisms of the Human Mouth. Archovey: Tr. Internat. Med. Cong., Lond., i, 862. 
Independent of the findings of Black, Miller and Archovey, there are indications that pyorrhea is a parasitic disease, since almost always persons coming in close contact with an infected individual, as man and wife, contract it. Children of parents having pyorrhea, when they become adults, are very subject to the disease. This is by no means a positive indication that the disease is infectious, but is suggestive.

The great variety of organisms found in the pockets about teeth affected with pyorrhea alveolaris renders the task of isolation exceedingly difficult.

Owing to the temporary closure of pyorrhea pockets about teeth on extension of infection, an acute condition is frequently observed. It simulates alveolar abscess and is often mistaken for that disease. During this stage opportunity is afforded for the greater absorption of bacterial poisons and the escape of bacteria into the lymph and blood streams. It is to these causes that we should most commonly expect systemic participation from pyorrhea, rather than from the swallowing of pus liberated from pyorrhea pockets; we cannot, however, ignore the danger to patients from the continual swallowing of this pus. True pyorrhea is very intractable to treatment. It may be cured if taken in time, and remain cured, provided the cooperation of the patient is elicited to carry out proper hygienic measures afterward.

With few exceptions the attitude of the profession toward pyorrhea alveolaris, as in chronic alveolar abscess, has been one of indifference. That it should have been supposed that such a disease may exist indefinitely and not be productive of evil is surprising.

\section{Osler says: ${ }^{8}$}

Of the twenty cases of pernicious anemia which I had under observation in 1909 , pyorrhea alveolaris was present in more than half. Certain types of nephritis are also believed to be due to oral infection.

In the majority of extensive cases of pyorrhea alveolaris, much purulent matter is discharged into the mouth. Most of it goes direct to the stomach, while some of the bacteria and their products which have not been discharged into the mouth may pass from the granulating walls of the pus pockets, through the lymph and blood channels, to all parts of the body. A question of paramount importance now is: What effect do these poisons have on the system and what are their physical indications?

22 East Washington Street.

8. Osler: Practice of Medicine, p. 440. 